\title{
Reorganisation of Oncologic Care in Greece: A Proposal
}

\author{
Christos Emmanouilides* MD, PhD \\ Interbalkan Medical Center \\ Asklipiou 10, 57001 Pylaia, Greece
}

Received 5 November 2015; Accepted 14 January 2016

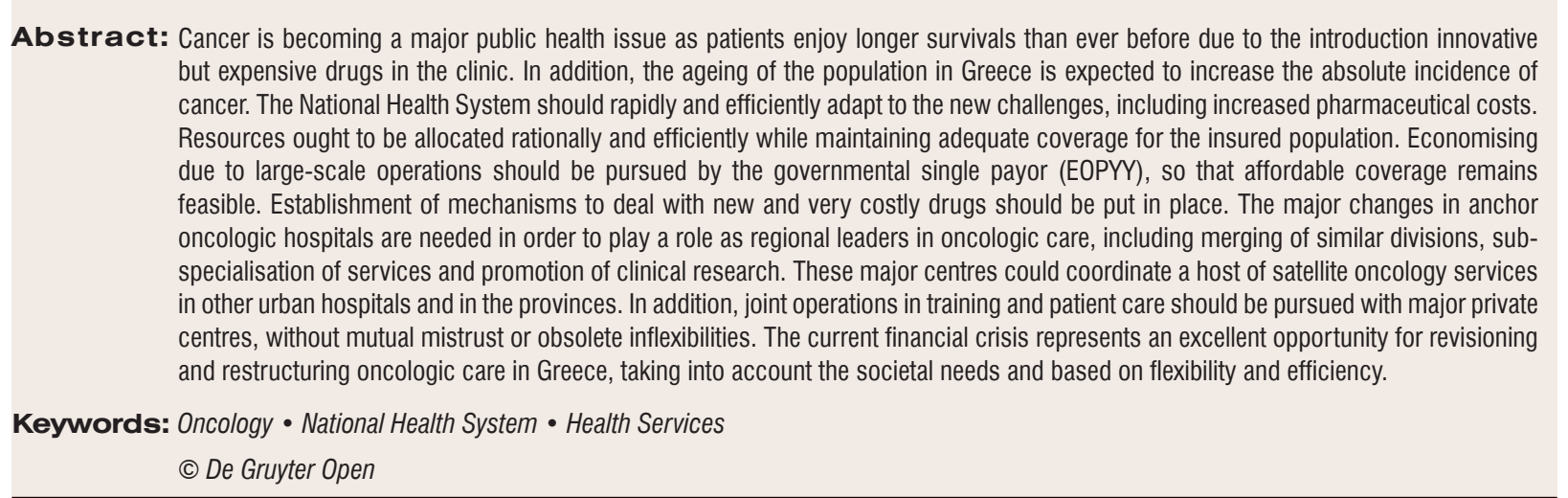

\section{Introduction - The financial aspect of cancer}

Cancer has become the primary cause of death worldwide. This is due to many factors, depending on the epidemiology of each region. In Greece, ageing of the population, as cancer is more common in older age, as well as the deterioration in lifestyle in recent decades, are considered as the contributing factors. The increase in incidence is not the only epidemiological but ultimately a fiscal problem. The survival of cancer patients has considerably lengthened, leading to an increased prevalence of the disease, long-term morbidity and thus a higher economic burden on society. Moreover, the introduction of many new, but extremely expensive, drugs has exponentially boosted the pharmaceutical cost, which has already preoccupied advanced societies. It is not generally uncommon for newer drugs to cost over 3000 euros monthly. $(1,2)$ The rising costs of diagnostic tests, e.g. molecular analysis, imaging methods and precision radiotherapy methods, must be added to the increased health care costs. (3)

In Greece, the incidence of cancer rose from 37,000 new cases in 1998 , to 41,000 in 2012 with 28,500 deaths.(4) Unfortunately, in men the most frequent tumour that is refractory to treatment was lung cancer (24\% of cases), as opposed to prostate cancer in the rest of the European Union (4). Public expenditure on health in 2012 amounted to $6.2 \%$ of the gross domestic product (GDP: 193.7 billion euros), while an additional $2.9 \%$ involved private spending. Rough estimates, based on disease coding system (ICD coding), show that in 2012, public spending on tumours was approximately $13 \%$ of all-health expenditure, i.e. $0.86 \%$ of GDP or 1.66 billion. This expenditure is expected to increase due to the above-mentioned reasons.(5) Hence, it can be calculated that in 2012 the average public expenditure per patient with cancer diagnosis was 40,500 euros. And this huge amount for the average Greek does not include fixed costs for staff and infrastructure or losses to the 
economy by reduced productivity and loss of workhours of victims or relatives.

Concerns about rising costs of oncologic health systems are expressed in all Western societies. In the United States, medical costs rose sharply from 5\% of GDP in 1965 to $20 \%$ in 2014.(6). In 2005, the total oncology costs amounted to 210 billion US dollars (1.5\% of GDP). It is estimated that the cost will double by 2030 . The direct costs (medical expenses and medications) are expected to increase from 124 billion in 2010 to 204 billion in 2020. (7)

European states have mainly public health systems and are more keen on maintaining existing free access status. Generally, health expenditure amounts to $9-10 \%$ of GDP in European countries (except: less in the United Kingdom at $6.5 \%$ ), of which $2-3 \%$ relate to private expenses. In the last decade, oncological costs represented $6-10 \%$ of these expenses. (8) The introduction of new expensive drugs has created rigorous control systems with restrictions that vary by country. The strictest system in Europe, the NICE of the United Kingdom (UK), implemented domestic evaluation of costeffectiveness assessments of all new drugs; as a result British patients, not infrequently, do not have access to drugs that are available to other Europeans. Despite these limitations, the overall oncological cost averages $£ 42,400$ for each of the 50,000 newly diagnosed cancer patients annually, clearly not a trivial amount.

But even in the UK, revision of the open access policy has been proposed. This is a challenge to the main core premise of the system, which now seems unsustainable. (9) Specifically, it has been suggested that the necessary additional funding to the soaring needs of the health system should come from the financial participation of the patient, as a preferred method rather than increased taxation. (9) In fact, various studies have shown that due to the inflexibility of the need for oncological care, patients agree to participate in the costs in order to ensure proper treatment. $(10,11)$ Greek patients essentially do the same by providing underthe-table payments in public hospitals or resorting to the private sector. The French health system, more akin to the Greek because, while based primarily in the public sector, incorporates large private participation, provisions significant co-payments at varied rate for almost all services including pharmaceutical acquisition and has imposed quantitative restrictions on offered services to prevent excesses.(12) Therefore it appears that participation of the patient in health expenditure within the public system is a necessity for the survival of the system itself. Naturally, the state should provide special assistance to vulnerable social groups.
Perhaps all the above calculations are moderate, because the discovery of new drugs increases the median survival of cancer patients at a heightened and excessive cost. For example, twenty years ago, a patient with colon cancer could only be treated with fluorouracil with median survival of 7.6 months and overall pharmaceutical costs under $\$ 100$. Nowadays, a similar patient enjoys a median survival of about 2 years with pharmaceutical costs of approximately $\$ 250,000$ per year. (6) Such developments could not have been foreseen by health economists only a couple of years ago.

Various systems have been developed to address the high cost of drugs. In the US, patients have a $25 \%$ co-payment to the cost of medicines, thereby restricting access to expensive drugs, unless a private insurance exists for offsetting cost. At the same time, bankruptcies for health reasons have increased. In the UK, new expensive drugs are covered only when they do not cost more than 50,000 per QALY (quality of life -year). Thus, in UK, an entire economic sector has been developed for assessing pharmaceutical costs; the value of a new drug is based on the benefit to cost ratio compared to previous treatments (incremental cost efficacy ratio [ICER]).

Therefore, all these factors, together with the legitimate demand of the population of a well-governed state for assured but rational care, necessitate the operation of a modern, intelligent and flexible oncology health system in Greece. A system that optimises the performance and delivery of health services, in compliance with the requirement of the times to avoid unnecessary costs is needed, with adaptability to the constantly changing realities in the field of oncology. Moreover, this system should protect patients and, which is very important, relatives from unnecessary suffering and loss of productive time.(13) Thoughts that relate to this aim are presented below, hoping to incite a productive public debate about this issue.

\section{Establishing an oncology national committee}

A central planning body of oncological policy should have a significant say on decision-making, with overriding power over governmental decisions. This will avoid the arbitrariness of the central government and better control any impulsive edicts by the State. The committee should be composed of state representatives, oncologic and academic scientists and social partners, as the society is ultimately the party directly concerned. There must 
be at least representatives of the following organisations (suggested): Ministries of Health, Economy, Welfare; National Health System (EOPYY), Medical Oncology Association (EOPE), managers of Public and Private Hospitals, Hellenic Medical Association, Association of Pharmacists, Academic Oncologists, Professors of Public Health, Associations of Cancer Patients, Consumers' Associations, Chamber of Commerce. All government decisions pertaining to oncology must be approved by the above Committee before given for voting in the Parliament or issued as circulars. If a governmental proposal is rejected by the Committee, a minister or representative could present and support the proposal. If consent could not be achieved, then only the prime minister could take responsibility for the decision. Members of the committee may discuss any matter related to oncology policy and should propose any improvements. The Committee may invite or assign issues to experts when necessary.

\section{Oncology map}

Establishing a thorough oncological map aims at identifying the oncological needs by region (county or district) and thus facilitate the subsequent allocation of oncological services. In this context, the availability of the private sector should be taken into account for meeting the needs. The oncological services can be divided into simple (e.g. administration of postoperative chemotherapy) or complex (e.g. radiotherapy, concurrent treatments, bowel surgery) or specialised (e.g. stereotactic radiotherapy, interventional oncology, specialised surgeries such as colon, pancreatic, brain, lung etc.).

Within this framework, expertise centres can be founded (centres of excellence) for specialised multidisciplinary treatments, for conditions such as cancer of the rectum, stomach, pancreas, lung, sarcomas and glioblastomas. The reason for the creation of these centres is that the results of operations are in direct relation with expertise and the number of operations carried out, aided by the function of interdisciplinary tumor boards. These centres will be staffed, partially at least, by surgical oncologists. Regional patients should be diverted to such centres.

\section{Public hospitals and clinics}

The public oncology system should be structured around Oncology Reference Centres (ORC), which will coordinate peripheral oncological functions. Cancer hospitals, academic oncology departments or major oncology departments should operate as ORC. The system will pursue decentralisation of simple oncology activities and optimise the operation of ORCs, alleviated from the burden of trivial cases.

It is desirable to establish positions for oncologists in non-oncology hospitals such as urban hospitals but especially in the provinces. The establishment of such positions in provincial hospitals is essential to address the unequal distribution of oncologists in favour of big cities (14). These oncologists participate in the activities of the hospital as consultants, but they should also be capable of providing simple oncological care. They will be in direct contact and cooperation with the major cancer hospital/oncology department in their region or the university oncology clinic (ORC). They will participate in mandatory educational activities of the ORC as well as in tumour boards via teleconference. They are obliged to continue treatment of patients diagnosed or starting treatment at ORCs, always in close cooperation. They will also participate in the research activities of the ORCs, functioning as satellite clinics, with support from the ORCs.

The cancer hospitals and the university clinics, where these are lacking, are crucial in supporting Greek oncology and should be upgraded to fulfill their role. Having multiple medical oncology or surgical divisions in the same cancer hospital is unproductive. Merging such divisions into one larger unit under a unified management creating a larger sector will be more efficient in many ways. This will enable specialised sections to be set up within the large department of oncology, according to organ systems, i.e. respiratory, digestive, gynaecological, urinary, and others. These sections will work closely within themselves and with other surgical specialties and will coordinate clinical and scientific activities. All chemotherapeutic treatment will be performed by medical oncologists. Other disciplines will cooperate on diagnosis, treatments and interventions but will cease to prescribe pharmaceutical anti-tumor treatment.

Cancer hospitals, as discussed above, will collaborate with other oncologists in other urban or provincial hospitals in their area, which would continue treatments initiated at the oncological hospital. The continuation of treatment could also be undertaken by the private sector. Peripheral oncologists will provide feedback to the main hospital. The alleviation of cancer hospitals from the time and expense of ordinary treatments (e.g. maintenance therapy with a harmless monoclonal antibody every 21 days for a year) will allow the allocation of resources, time and effort to complicated medical problems, teaching, research 
or other productive activities. Thus a modernised cancer centre would easily achieve excellence and it would attract not only patients but also clinical studies, resulting in many benefits as discussed below.

\section{Cooperation with private oncology}

Private medicine in Greece is a reality that cannot be ignored. A clever public health system should take advantage of its existence rather than compete with it. The public health system would save resources if it ceded simple oncological treatments to the private sector (outsourcing) mainly due to staff-related cost savings. It could therefore be contracted with private doctors or clinics, ensuring that public patients have fully covered hospitalisation in the private sector, even at lower prices. This, of course, requires a reliable EOPYY that will come up with the proper payment in due time. Patients of the public system who would elect this course would benefit from a relatively less busy treatment area day-care type, without intermingling with the severely ill. Furthermore, simple oncological treatments could take place in a well-equipped private outpatient office, with availability of appropriate infrastructure and personnel, thus circumventing the need for hospitalisation, as done in several countries. The EOPYY could contract with such centres of 'primary' oncology in order to better serve the insured and enjoy savings in expenses and personnel.

\section{The role of the national health service provider (EOPYY)}

The current status separates oncology drugs into two categories, the common ones, distributed by pharmacies, and the nosocomial ones. The latter generally include the more expensive drugs and are distributed by EOPYY public pharmacies and less commonly by public hospitals. This system enables the state to negotiate with pharmaceutical companies better prices for 'expensive' drugs whose patent has expired, due to competition. Apparently, EOPYY has not managed so far to reduce the purchase price of expensive drugs with active patent protection.

Most oncology drugs are nosocomial. In order for the patient to procure such drugs, a complicated path must be followed. First, he is admitted to a hospital or private clinic, then a hospital prescription is issued and in the same day he, or (most commonly) a relative, must go to the EOPYY pharmacy (which can be located several kilometres away) to execute the recipe and bring the drug to be administered. In other words, an admitted, perhaps sickly, patient ought to leave the hospital and return with the drug to be given. So much time and productivity wasted, so much suffering, discomfort, travel expense and delay should not be satisfactory. It should be noted that the reason for which the major private clinics do not provide nosocomial chemotherapeutics is the justified mistrust about timely reimbursement by EOPYY.

The ability of EOPYY to be supplied drugs via tenders at better prices is unquestionable. Therefore the current system is justified in a sense, but the whole process could be rationalized in order to avoid troubling patients. For instance, procurement of the drugs could take place before hospitalisation, perhaps with an obligation to be administered within a week. The existence of electronic prescriptions is a sufficient means to avoid pernicious prescribing. In addition, a more efficient geographical distribution of dispensing points would reduce long routes often travelled by the patient or his attendants to reach the single regional EOPYY pharmacy. It must be considered unacceptable for oncologic care to depend on the availability of relatives to pick up the drug from the EOPYY pharmacy that may be located kilometres away.

Moreover, many common drugs of the nosocomial type are now affordable (doxorubicin, paclitaxel, docetaxel, irinotecan, oxaliplatin, zoledronic acid, capecitabine). Thus, their distribution could be liberalised with little direct added cost to the system. No admission should be mandated for many of those drugs that could easily be administered in an outpatient setting. (15) This would result in significant reduction of bureaucracy and personnel resources, thus direct and indirect cost savings. If the above are implemented, a patient receiving an antibody administered over thirty minutes would not have to spend a whole morning in a hospital occupying the bed, while waiting for the hospital prescription to be issued and until his drug is brought by his companion.

Another important issue is the lack of coverage of entirely appropriate chemotherapeutic drugs due to absence of formal indication by the National Pharmaceuticals Organization (EOF). This is mainly because pharmaceutical companies wished to gain regulatory approval only for common types of cancer, in order to ensure fast and broad marketing. As a result, the formal indications of a drug are a subset of the cancers where it is active. For example, there is no drug approved for cholangiocarcinoma, cancer of the nasopharynx, oesophagous, smal cell cancer of the prostate etc. It is proposed that reimbursement is granted for drugs, especially for inexpensive ones (e.g. below 100 euros per dose) according to the existing guidelines of the Hellenic Society of Medical Oncology (HESMO). 


\section{Drug coverage}

The issue of financial coverage of drugs is contentious in any society and is governed by various policies in different countries. In the US, there is usually a significant participation of the insured at a $25 \%$ rate, which renders modern drugs inaccessible by most, whereas in the UK, where access is free, many approved drugs are not administered by the public health system for financial reasons. Other European countries maintain free access to expensive drugs but with many restrictions; in some instances, prices are reduced by negotiations with pharmaceutical companies, or they are covered only if there is a clinical effect.

With the already started and imminently intesifying introduction of very expensive medicinal products, the existing system of free access to medicines cannot be maintained. Some form of patient involvement or some kind of restriction must be established in order to preserve the sustainability of the health system. A generous health system, which in the end is unable to pay is worse than a neat, less generous perhaps, but credible system. General principles are proposed below:

Non-hospital drugs, which are the most economical, can be provided with some patient participation (e.g. $10 \%$ ) as medicines for other diseases. This will save resources available to cover the very expensive drugs. Currently nosocomial but off-patent drugs could be transferred to the non-nosocomial category, as prices are already reduced due to competition.

The nosocomial and expensive drugs can be tiered according to evaluation of their cost-benefit ratio. The National Oncology Committee could oversee their categorisation into: (a) medications that increase the cure (absolutely necessary), (b) have a favourable cost-benefit ratio and (c) high cost-benefit ratio. The first category may be provided with a minimal charge to the patient (e.g. $5 \%$ or 20 euro per dose), the second with somewhat larger (e.g. $10 \%$ or 40 euro) and the third at higher participation (20\% or 100 euros), with provision for impoverished patients (numbers are only indicative).

Finally, EOPYY retains the right to apply restriction criteria for granting very expensive drugs, with right of appeal to a special committee. Special committees will also consider special requests, e.g. hospitalisation abroad, treatments with imported medicines, special examinations etc.

Instituting even a small patient financial participation would limit excessive use of expensive drugs and would make the patient involved in the decisionmaking with shared responsibility. All such measures should be debated and ultimately adopted by the National Oncology Committee. Moreover EOPYY needs to increase its ability to extract lower prices from current or future drugs. The only way to achieve this is to reserve the right not to cover a remedy or to threaten to put in a list of high patient co-payment, thus pushing pharmaceutical companies to reduce prices.

\section{Treatment and support of terminal patients}

In every developed society, the care of terminal patients in the last months of life when cancer treatment ceases to have meaning is the subject of an organised network of specialised services at home (hospice care). This approach ensures better quality of life and provides services more suited to the real needs of the patient, while offering great savings by preventing unnecessary hospitalisations. The terminal treatment system is based on specialised paramedical staff under the overall guidance of doctors and covers not only medical needs, but also social and psychological needs. Such systems must necessarily develop in big cities and work with non-specific home-care services in smaller towns. The expenditure should be covered by EOPYY, as it offers savings.

\section{Oncology training}

The proper training of trainees in oncology should be a concern of the health system. The current system confines the trainee in the narrow context of a specific oncologic division/clinic. Oftentimes the trainee spends much time on procedural and bureaucratic chores of considerable workload instead of focusing on education.

More attention should be paid to the education of young oncologists by the system; the issue is too important to be left to the goodwill of individual directors. A young oncologist-to-be should be exposed to different approaches and different ways of thinking in order to mature as a professional personality. Currently, each trainee 'belongs' to a certain division/clinic. After the proposed restructuring of cancer hospitals, all residents will belong to the major oncology department with mandatory rotation within the department to all organsystem sections.

Also, trainees should have a semester at strictly selected, cooperating and controlled private oncology centres. Large private centres involve highly developed 
practices with modern oncology facilities. Trainees exposed to these centres would only benefit. They will experience a possibly different patient population, a different practicing style, hence increasing their oncologic input and achieving a more comprehensive understanding of the field. Private centres participating in training programs must provide clear educational activities and supportive environment for junior oncologists. They should be assessed periodically by the ORCs for their educational adequacy and they should ultimately be approved by National Oncology Committee.

\section{Onco-nurses}

In oncology centres, nurses develop special skills and accumulate a huge experience that should be utilised for a more expedient and effective patient care. The institution of onco-nurse status must be recognised and promoted. The role of well-trained nursing staff should be upgraded above the level of ordinary nurse so that they may function as physician's assistants, able to lawfully solve simple medical problems with autonomy. Special training, seminars and title acquisition tests can formulate a specific curriculum and passing exams will be a requirement for obtaining the title. Instituting this upgrade would foster eagerness for learning, boost the morale of hard working nurses and serve as an appropriate recognition of their important contribution to cancer care. Moreover, Greece is almost the worst of OECD countries in the number of nurses with 3.2 per 1000 inhabitants, while the average is 9.6 and 5.1 in Portugal (OECD 2013). It has been shown that adequate nursing staff increases patient throughput and by shortening hospitalisation results in cost savings.

\section{Compensations}

Any system aspiring to be successful must be staffed by satisfied personnel that enjoys their work. Employees in the health system toil much while they usually feel that their efforts are not recognised. Open-minded and forward-looking executives without political party dependencies and with related studies in health administration are needed as hospital managers.

It is of paramount importance that financial grudges are eliminated. Immediate payment of all accrued on call duties and adequate staffing are essential. The use of evening clinics within hospitals and longer operation room hours should be generalised in order to make more efficient use of the infrastructure and generate income for the hospitals.
The interest of the hospital managers for all staff should be apparent and substantial. Programmes addressing employment burnout and all kinds of scientific, artistic or social events that disrupt daily routine and tighten relationships must be pursued. Nurseries at the workplace would facilitate staff. Moral and material rewards at the extent possible would create incentives for excellence. The environment of those who work by the oncology patient should generally comfort the heavy emotional and physical burden of the daily confrontation with cancer.

\section{Clinical research}

Clinical research benefits in many respects. Firstly, patients gain access to new drugs before they become commercially available. Participants often enjoy free clinic visits, tests and medications. Clinical research can also be a reason for capital inflow to the country, as studies are financed from abroad. Most importantly, advances in oncology are predominantly based on clinical research; participation in clinical research familiarises Greek oncologists with the newest developments, updates their knowledge and fosters foreign collaborations. Therefore clinical research should be pursued and supported in every way.

The reorganisation of cancer hospitals into sectors per organ system will make them attractive as sites for clinical research, provided that this is supported by the hospital management. A smart public system should aim at increasing clinical research. A collateral benefit would be the creation of research-related jobs. Fully manned clinical research operations would be even more attractive to the pharmaceutical industry, hence bringing more studies and ultimately generating more available funds. As mentioned above, as many tests are provided by the clinical study, further savings for the public system are realised.

A forward-looking cooperative spirit, without squabbling and selfishness, would allow movement of patients within a wider geographical area of the public and private health system for inclusion in relevant clinical trials. Such studies should be publicised in order to attract patients, not only to the medical community but also to the community at large. By developing a clinical research culture, all parties involved have something to gain: patients improve their care, hospitals reduce expenses, the government generates income and finally doctors feel actively involved in the scientific advances of their field. 


\section{Conclusion}

The rapid changes in oncology and the related pharmaceutical costs outpace the ability of health systems to cope with the situation. It is mandatory for National Health System in Greece to adapt to the new environment in the most efficient way. Dogmatic attitudes of the past ought to be abandoned in order for the health system to survive in the times of fiscal crisis. The sustainability of the health system as it pertains to oncology requires incorporation of modern hospital management, flexibility, cooperation, utilisation of a thriving but regulated private sector and involvement

\section{References}

[1] COLLIER R. Drug development cost estimates hard to swallow. Canadian Med Assoc J, 2009, 180:279280

[2] DIMASI JA, GRABOWSKI HG. Economics of new oncology drug development. J Clin Oncol 2007, 25:209-216

[3] КАРАМПАН Е, ХАТZАКН А, ПОАYZO $N$,

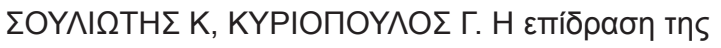

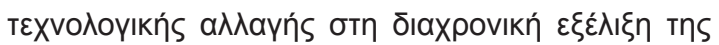

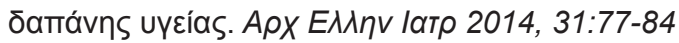

[4] WHO-International Agency for Research on Cancer. http://eco.iarc.fr/EUCAN/ Country. aspx?/SOCountryCd=300

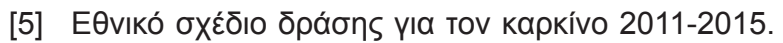

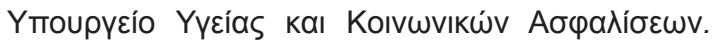
http://www.anticancer.gov.gr SxedioDrasis_24selido.pdf

[6] MEROPOL NJ, SCHULMAN KA. Cost of cancer care:issues and implications. J Clin Oncol 2007, 25:180-186

[7] MARIOTTO AB, YABROFF KR, SHAO Y, FEUER EJ, BROWN ML. Projection of the cost of cancer care in the United States: 2010-2020. J Natl Cancer Inst 2011, 103:117-128

[8] SULLIVAN R, PEPPERCORN J, SIKORA K, ZALCBERG J, MEROPOL NJ, AMIR E ET AL. Delivering affordable cancer care in high-income countries. Lancet Oncol 2011, 12:993-980 of societal partners in decision making. EOPYY should take advantage of its bargaining power to ensure affordability, ease of access and rational allocation of available funds. Responsibility for major decision should be taken away from the political governments and should be transferred to a pluralistic National Oncology Committee as described above. Major cancer hospital reorganisation is necessary to lead Greek oncology to the new era. All these should take place with the scope of providing a reliable, fair, sustainable and humane health system to our fellow citizens, whom we are to serve.

[9] AGGRAWAL A. Affordability of cancer care in the UK-Is it time to introduce user changes? J Cancer Policy 2014, 2:31-39

[10] LANG HC. Willingness to pay for lung cancer treatment. Value in Health. Journal of the International Society for Pharmacoeconomics and Outcomes Research 2010, 13:743-749

[11] WONGYN, HAMILTONO,EGELSTONB, SALADOR K, MURPHY C, MEROPOL NJ. Understanding how out-of-pocket expenses, treatment value, and patient characteristics influence treatment choices. Oncologist 2010, 15:566-576

[12] CHEVREUL K, DURAND-ZALESKI I, BAHRAMI SB, HERNANDEZ-QUEVEDO C, MLADOVSKY P. France Health System Review. Health Syst Trans 2010, 12:1-291

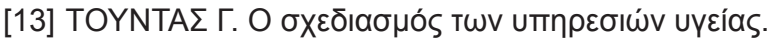

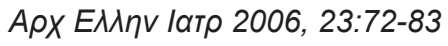

[14] $\Sigma O Y \wedge I \Omega T H \Sigma \mathrm{K}, \mathrm{A \Theta ANA \Sigma AKH \Sigma} \mathrm{K,} \mathrm{ГKO \wedge NA} \mathrm{X,}$

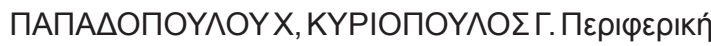

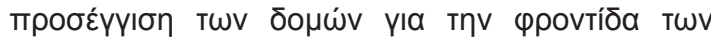

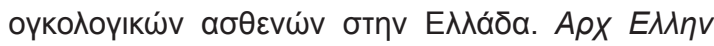
Іатр 2009, 26:808-817

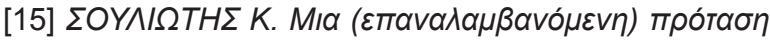

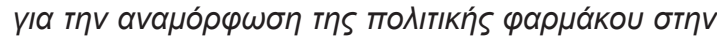

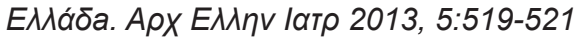

